\title{
SEARCH FOR A DRUG-CANDIDATE FOR THE SECOND GENERATION OF POLYENE ANTIBIOTICS
}

\section{A.N. Tevyashova, E.N. Bychkova, N.E. Grammatikova, E.P. Pereverzeva}

Laboratory of Chemical Transformation of Antibiotics, Gause Institute of New Antibiotics, 119021, Russia, Moscow, B. Pirogovskaya, 11.

DOI: 10.19163/MedChemRussia2021-2021-146

E-mail:chulis@mail.ru

Amphotericin B (AmB, Fig. 1) is the drug of choice for treating the most serious systemic fungal or protozoan infections. Nevertheless, its application is limited by low solubility in aqueous media and serious side effects such as infusion-related reactions, hemolytic toxicity, and nephrotoxicity. Owing to these limitations, it is essential to search for the polyene derivatives with better chemotherapeutic properties. With an objective of obtaining AmB derivatives with lower self-aggregation and improved solubility, we synthesized a series of amides of AmB bearing an additional basic group in the introduced residue. The screening of antifungal activity in vitro revealed that $\mathrm{N}$-(2-aminoethyl)amide of AmB (Amphamide, Fig. 1) had a superior antifungal activity compared to that of the paternal AmB. Preclinical studies in mice confirmed that Amphamide had a much lower acute toxicity and higher antifungal efficacy in the model of mice candidosis sepsis compared with that of AmB [1]. Thus, discovered Amphamide is a promising drug-candidate for the second generation of polyene antibiotics and is also perspective for in-depth preclinical and clinical evaluation.

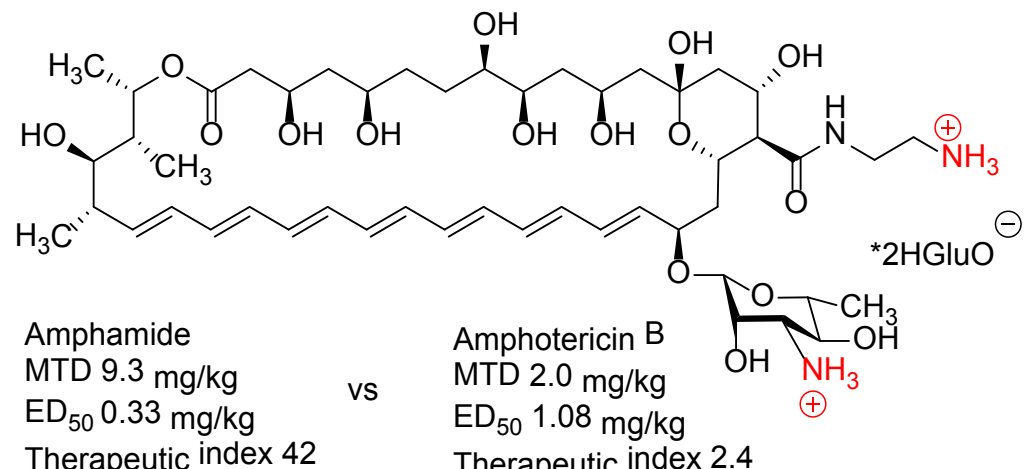

Study of chemical modification of AmB was funded by RFBR, project number 20-04-00467.

\section{References}

[1] A.N. Tevyashova et. al., ACS Infect. Dis. 2020, 6, 2029-2044.

https://dx.doi.org/10.1021/acsinfecdis.0c00068. 